Le rôle des collectivités locales dans la gouvernance alimentaire : le cas du projet alimentaire territorial de ToursMétropole-Val-de-Loire

\author{
José Serrano \\ UMR 7324 Citères \\ Université de Tours \\ 33 allée F. de Lesseps \\ 37200 Tours \\ jose.serrano@univ-tours.fr
}

\author{
Céline Tanguay \\ UMR 7324 Citères \\ Agence d'urbanisme de l'agglomération tourangelle \\ 56 avenue Marcel Dassault \\ 37200 Tours \\ tanguay@atu37.fr \\ Jean-Louis Yengué \\ EA 2252 RURALITES \\ Université de Poitiers \\ MSHS, Bâtiment A5 \\ 5, rue Théodore-Lefèbvre \\ TSA 21103 \\ 86073 POITIERS Cedex 9 \\ jean.louis.yengue@univ-poitiers.fr
}




\section{Le rôle des collectivités locales dans la gouvernance alimentaire : le cas du projet alimentaire territorial de Tours- Métropole-Val-de-Loire}

Résumé :

La loi d'avenir pour l'agriculture l'alimentation et la forêt de 2014 vise à favoriser une relocalisation de la production alimentaire à l'échelle locale en mettant l'accent sur le rôle des acteurs locaux. Elle propose comme dispositif les projets alimentaires territoriaux pour coordonner leur action selon une démarche de projet. La loi donne aux collectivités locales un rôle essentiel dans la gouvernance des acteurs. Dans cet article nous allons questionner une démarche de relocalisation alimentaire en étudiant le projet alimentaire territorial de Tours Métropole Val de Loire. À défaut d'une démarche transversale, nous montrerons que Tours Métropole adopte une démarche techniciste qui conduit au maintien d'une prédominance de acteurs des filières longues dans le système alimentaire local.

Mots clés : projet alimentaire territorial, métropole Tours-Val-de-Loire, relocalisation production agricole, circuit alimentaire de proximité

\section{The role of local authorities in food governance: the case of the Tours-Métropole-Val-de-Loire territorial food project}

Abstract :

The 2014 law on the future of agriculture, food and forestry aims to promote the relocation of food production to the local level by emphasising the role of local actors. It proposes territorial food projects as a mechanism to coordinate their action according to a project based approach. The law gives local authorities an essential role in the governance of stakeholders. In this article we are going to look at a food relocation approach by studying the Tours Métropole Val de Loire territorial food project. In the absence of a transversal approach, we will show that Tours Métropole adopts a technical approach which leads to the maintenance of a predominance of players from the long supply chains in the local food system.

Key words : territorial food project, Tours-Val-de-Loire metropolis, relocalization of agricultural production, local supply of food products

Nomenclature JEL :

Q01 Sustainable Development

Q180 Agricultural Policy; Food Policy

R580 Regional Development Planning and Policy 


\section{Introduction}

La modernisation de l'agriculture a entraîné une séparation de la production agricole et de la fourniture de produits alimentaires. Les agriculteurs se sont spécialisés dans la fourniture de produits agricoles qui sont devenus des matières premières pour une industrie alimentaire (Bonny, 2005). Cette séparation est remise en cause par le regain d'intérêt pour une alimentation saine et de proximité à travers notamment les circuits courts (Lamine et Chiffoleau, 2012). Néanmoins, reconnecter production agricole et alimentation ne se résume pas à une réappropriation de la commercialisation des produits agricoles par les agriculteurs. De nouveaux acteurs interviennent dans l'organisation de la filière remettant en cause la logique d'intégration toujours plus poussée des exploitants dans des filières intensives et compétitives (Guiomar, 2012).

Ce texte s'intéresse à la relocalisation de la production alimentaire à travers les circuits de proximité et au rôle particulier joué par les collectivités locales. Les circuits de proximité sont définis par Praly et al (2014) comme des filières qui font intervenir des intermédiaires (transformation, distribution...) qui sont spatialement proches. Les circuits courts sont quant à eux définis par le nombre d'intermédiaires (zéro ou un) et traduisent une relation directe entre le consommateur et le producteur. Nous choisissons le concept de circuit de proximité qui est plus adapté à l'analyse de la relocalisation des filières alimentaires. Une partie importante de la littérature scientifique est consacrée à la gouvernance des circuits de proximité (voir notamment Perrin et Soulard, 2017, 2014). Est mise en avant la complexité du système alimentaire localisé du fait de la division des acteurs et de l'absence d'acteurs véritablement dominants ou structurants. Dans cet article, nous proposons d'analyser le rôle d'une collectivité territoriale à travers l'élaboration d'un projet alimentaire territorial (PAT).

La loi d'avenir pour l'agriculture, l'alimentation et la forêt (LAAF) du 13 octobre 2014 propose les PAT comme dispositifs pour favoriser les circuits de proximité et met en avant les collectivités locales comme chef de file pour la gouvernance alimentaire. Nous analysons le rôle de Tours-Métropole-Val-de-Loire qui décide en 2017 de mettre en place un PAT. La métropole tourangelle est emblématique des agglomérations françaises de taille moyenne présentant un fort contraste de densité humaine entre le noyau urbain et les espaces périurbains (Insee, 2011).

Nous ferons d'abord le point sur la reconfiguration du rapport au territoire des productions alimentaires localisées. Puis, nous présenterons l'action de la communauté d'agglomération de Tours(s)plus qui est devenue la métropole Tours-Val-de-Loire dont le projet alimentaire local prend la suite d'un schéma de cohérence territorial (SCOT) labellisé Grenelle et d'un plan climat air énergie territorial (PCAET). Nous analyserons le dispositif d'élaboration du PAT et son contenu. Dans une troisième partie, nous discuterons la portée de cette expérience pour une relocalisation de la production agricole. 


\section{Une gouvernance ouverte pour un projet alimentaire}

\subsection{Un cadre d'analyse centré sur la gouvernance}

La crise de confiance dans l'alimentation industrielle et la montée des enjeux environnementaux ont amené les consommateurs à consommer mieux, ce qu'ils ont traduit par la traçabilité et la recherche d'une proximité géographique et humaine (Delfosse, 2012). Ce regain d'intérêt pour la production alimentaire locale se développe dans un contexte politique et scientifique qui favorise les approches territoriales. Progressivement, les politiques publiques alimentaires insistent sur l'échelon local et la coordination des acteurs mettant ainsi en situation privilégiée les collectivités locales.

Les politiques alimentaires ont été développées au sortir de la seconde guerre mondiale et on peut distinguer trois phases (Montaigu, 2018) : une politique de sécurité alimentaire normative (1945-1980) liée à la production et à l'approvisionnement en denrées alimentaires, une politique axée sur les enjeux sanitaires et nutritionnels (19902000) et une politique plus territorialisée (à partir des années 2000) axée davantage sur la coordination des acteurs que sur les enjeux sanitaires. Dans ce cadre, les circuits courts sont présentés comme un moyen d'ancrer la production alimentaire localement et de coordonner les acteurs. Ils font l'objet de plans successifs. Le dernier en date est le plan alimentaire national de la LAAF du 13 octobre 2014 qui dans l'article 39 introduit les PAT.

Les PAT sont le fruit d'une co-construction par les acteurs territoriaux. Pour aider à leur réalisation la loi prévoit qu'ils « s'appuient sur un diagnostic partagé de l'agriculture et de l'alimentation du territoire et la définition d'actions opérationnelles visant la réalisation du projet $~$. L'objectif est de fédérer les différents acteurs d'un territoire autour de la question de l'alimentation, contribuant ainsi à la prise en compte des dimensions sociales, environnementales, économiques et de santé de ce territoire. La loi place les collectivités en chefs de file du projet alimentaire territorial ce qui rend important leur rôle de coordination.

Le programme national pour l'alimentation de 2000 décloisonne les politiques alimentaires. C'est un bouleversement des politiques alimentaires qui jusque-là traitaient les différentes dimensions de l'alimentation en silo (Creps, 2018). Or la prise en compte simultanée des différents thèmes auxquels peut être associée la question alimentaire (la filière alimentaire, l'économie, la gastronomie, la nutrition et la santé, l'alimentation pour tous, l'environnement et l'aménagement) nécessite une entente partagée sur les objectifs finaux et une coordination forte. .

En nous fondant sur une grille d'analyse élaborée par le réseau national pour un projet alimentaire territorial (RNPAT, 2018) ${ }^{2}$, nous avons étudié la démarche du PAT de la

\footnotetext{
${ }^{1}$ Loi n²014-1170 du 13 octobre 2014 d'avenir pour l'agriculture, l'alimentation et la forêt, article 39

2 Cette grille reprend une définition trasnversale de l'alimenation en six domaines et la démarche de projet telles que préconisées par la loi. Elle distingue les étapes classiques de la démarche de projet : diagnostic, programme d'actions et régulation des acteurs. Dans son analyse, le RNPAT donne un rôle central aux collectivités locales dans la gouvernance des PAT.
} 
métropole de Tours en observant le jeu d'acteurs dans le dispositif d'élaboration et en décryptant les décisions prises.

\subsection{Méthodologie}

Tours-Métropole-Val-de-Loire est à l'initiative d'un PAT lancé en 2017. Son comité de pilotage comporte 29 membres $^{3}$. Nous avons assisté au comité de pilotage du 21 février $2018^{4}$, réalisé sept entretiens semi-directifs ${ }^{5}$ et analysé les études réalisées pour éclairer le comité de pilotage (Agence d'Urbanisme de l'Agglomération de Tours 2017a et 2017b).

Les entretiens semi-directifs, menés auprès des acteurs les plus investis lors des débats, étaient destinés à approfondir les stratégies particulières des acteurs du comité de pilotage. Le guide d'entretien était divisé en quatre parties : le portrait et la description de l'activité de l'acteur, le rôle joué dans le comité de pilotage, la vision des objectifs du PAT, la perception et les relations avec les autres acteurs (Biais et Souchon, 2018).

Carte 1 périmètre du SCOT, Tours métropole et $15 \mathrm{~km}$

\footnotetext{
${ }^{3}$ Le groupe est constitué de 5 élus locaux, d'un grossiste, d'un agriculteur, de 3 membres ou techniciens d'Inpact 37, de 4 membres ou techniciens de la chambre d'agriculture 37, de 2 membres du Conseil de développement de Tours-Métropole, de 2 représentants du lycée agricole, de 4 membres de la SAFER, de 4 membres des services de la métropole, de 2 membres de l'agence d'urbanisme et d'une personne des services de la ville de Tours.

${ }^{4}$ Séance du 21 février 2018 de restitution de l'étude sur l'offre de production maraîchère conduite par la chambre d'agriculture 37.

${ }^{5}$ Conduits de février 2018 à juin 2018 auprès de deux agents du service économique de Tours métropole, de deux techniciens de la chambre d'agriculture 37, d'un maraîcher en agriculture biologique, d'un maraîcher conventionnel, d'un agent d'Inpact 37, d'un agent de l'agence d'urbanisme et du président du projet alimentaire territorial. Nous remercions Charlotte Biais et Coline Souchon étudiantes en 5 ième année de génie de l'aménagement et de l'environnement pour la réalisation et la transcription des entretiens.
} 


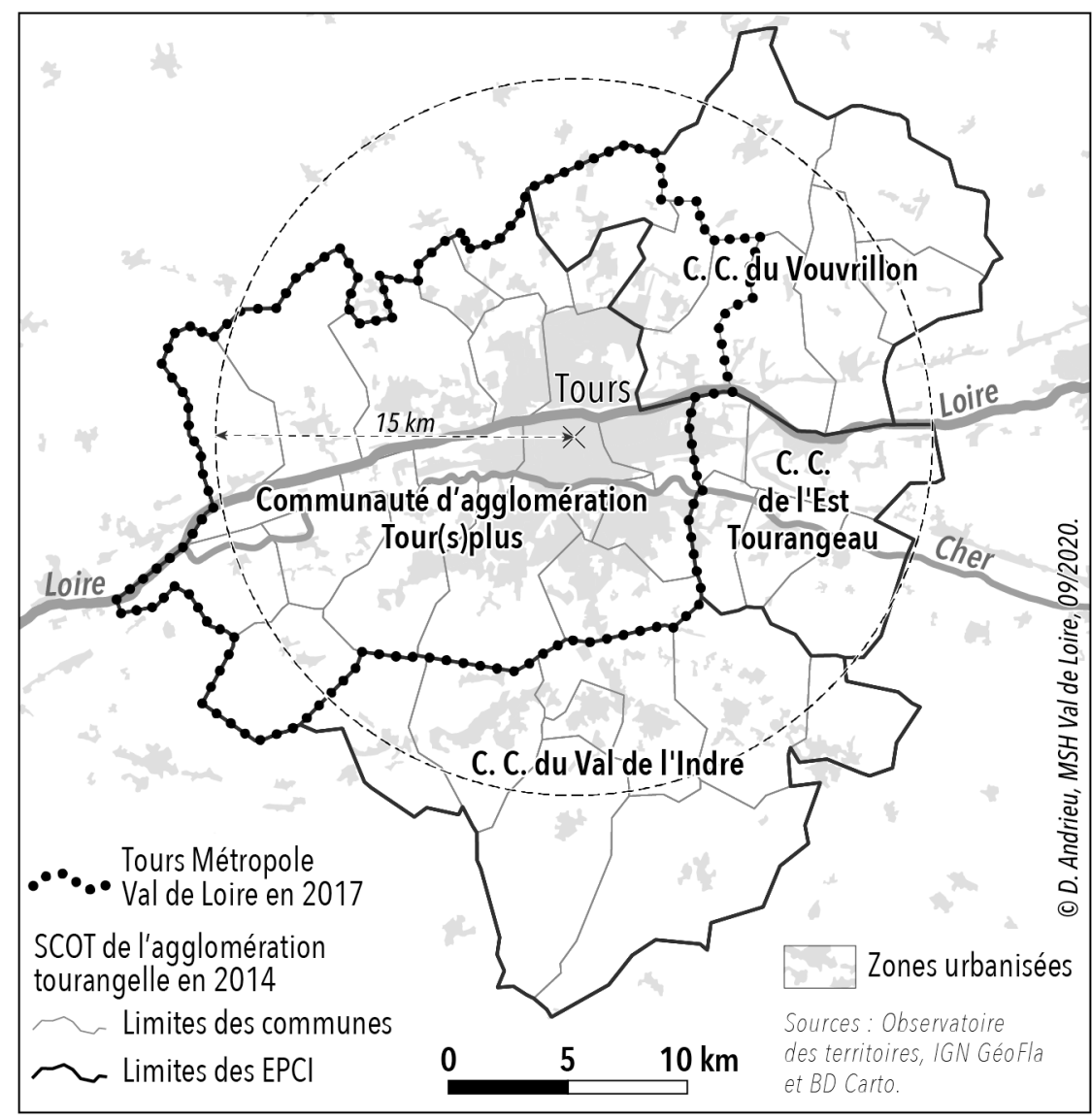

Le PAT succède aux réflexions menées sur l'agriculture périurbaine dans le cadre du SCOT en 2013 et du PCAET en 2011. Le PCAET a été conduit par la communauté d'agglomération de Tour(s)plus qui est devenue en 2017 Tours-Métropole-Val-de-Loire après avoir incorporé trois nouvelles communes (cf. carte 1). En 2013, le SCOT regroupait quatre intercommunalités dont la communauté d'agglomération de Tour(s)plus.

Le PAT a été officiellement lancé le 28 février 2017 par la lettre de mission du président de Tours-Métropole. Même s'il est encore en cours d'élaboration, l'essentiel de son contenu a été fixé au second semestre 2017 et en 2018 (cf. figure 3). C'est sur cette période que se focalisera notre recherche.

\section{Le Projet Alimentaire Territorial de la métropole Tours-Val-de-Loire}

Nous rappellerons les mesures en faveur de l'agriculture périurbaine du SCOT et du PCAET sous l'égide de l'agglomération tourangelle puis nous montrerons le jeu d'acteurs lors de l'élaboration du PAT.

\subsection{La prise de conscience progressive de la question agricole et alimentaire au} travers de l'élaboration de documents d'aménagement et environnementaux

$\mathrm{Au}$ cours de l'élaboration du SCOT de l'agglomération tourangelle, deux études ont permis de mettre en avant le "socle agronaturel" du territoire : "la trame verte et bleue" et "la caractérisation des secteurs à enjeux agricoles". Ces études sont présentées comme 
le fondement du premier pilier "la nature une valeur capitale" du plan d'aménagement et de développement durable du SCOT approuvé en 2013. Cette approche a été traduite spatialement par une priorité donnée au renouvellement urbain $(60 \%$ de la croissance démographique) sur l'extension (40\%) et par une localisation préférentielle des extensions dans la continuité du tissu urbain autour des bourgs $(500 \mathrm{~m})$ et des gares (1000 m) selon un modèle de ville polycentrique.

Figure 2 : Identification des secteurs de conflits entre la trame verte et bleue, l'agriculture et les zones d'urbanisation future (aire du SCOT de l'agglomération tourangelle), Agence d'urbanisme de l'agglomération tourangelle 2013

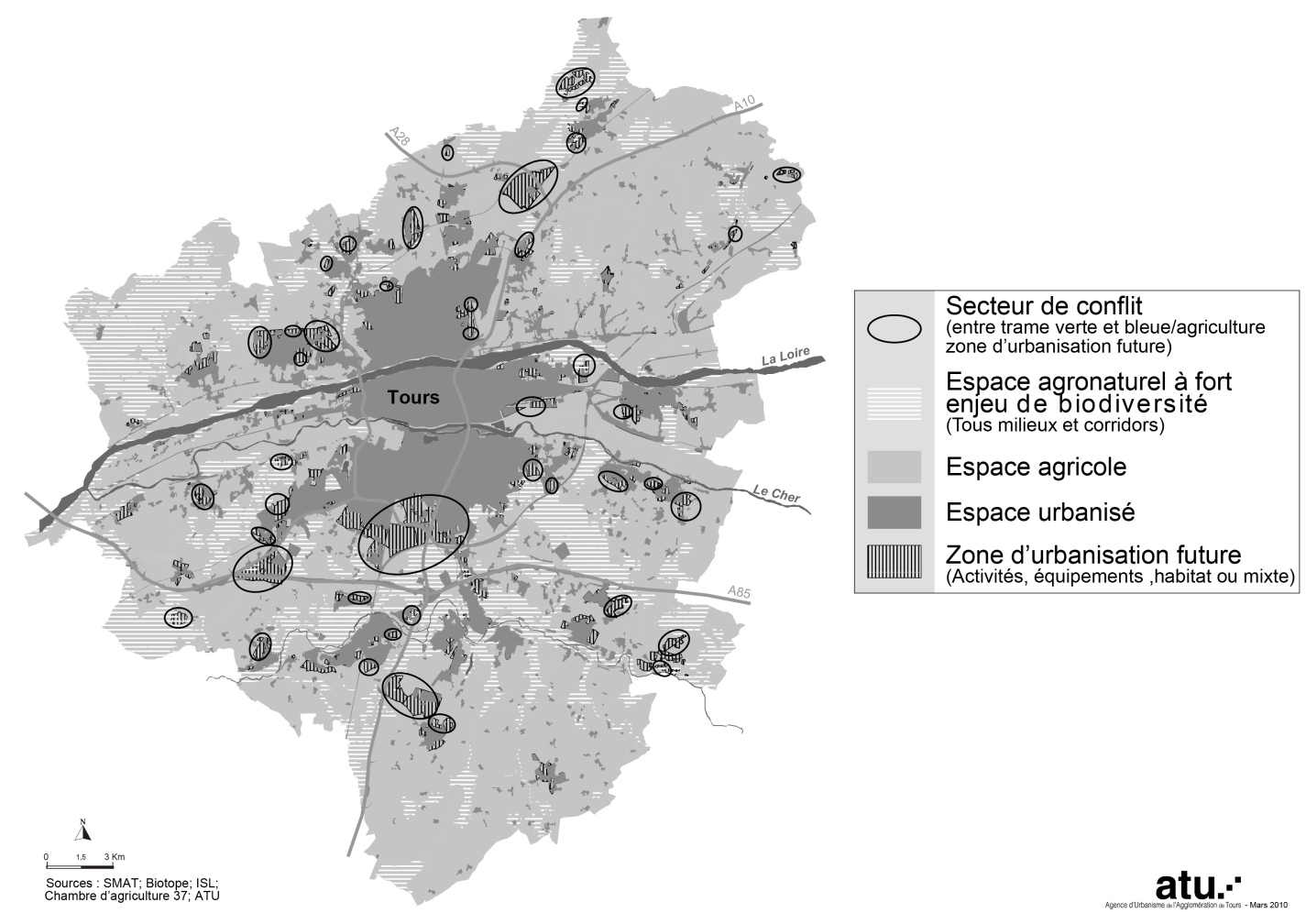

Les travaux, identifiant des espaces précieux pour les continuités écologiques ou agricoles, ont été croisés avec les zones d'urbanisation future dans les documents d'urbanisme en cours. Cette analyse a révélé des incompatibilités avec les ambitions de protection du "socle agronaturel" (cf. figure 2). Pendant le processus du SCOT, des réunions au sein des communautés de communes avec cartographie à l'appui ont permis aux élus de visualiser ces enjeux sur leurs territoires respectifs. Les discussions ont abouti à un déclassement de 400 ha sur les 1200 ha de zones à urbaniser inscrits dans les documents d'urbanisme en cours.

Le PCAET quant à lui, a été élaboré par l'ancienne communauté d'agglomération Tour(s)plus et approuvé en 2011. Il affichait dans son plan d'actions le développement d'une agriculture locale en trois points : soutenir une agriculture de proximité, installer des agriculteurs au cœur de la ville, faciliter les circuits courts.

En quatre ans, cette ambition a abouti à l'installation des trois maraîchers, tous localisées dans les zones inondables inconstructibles. Les futurs producteurs devaient 
répondre à un cahier des charges et déposer un dossier auprès de la communauté d'agglomération. Cette dernière n'exigeait pas au départ une production en agriculture biologique, mais les porteurs de projet devaient s'engager, dans un court terme suivant leur installation, à une production respectueuse de l'environnement. Ce que les porteurs de projets ont traduit par une conversion en agriculture biologique. D'autre part, il n'était pas demandé de répondre aux besoins locaux en termes de restauration collective dont les exigences étaient (et sont encore en termes de tonnage à produire) hors de portée de petits producteurs.

Si concrètement, on peut considérer que peu de choses ont été faites pour développer l'agriculture nourricière dans l'aire du SCOT, ces actions pointillistes ont eu le mérite de mettre "sur la table" le sujet d'une production alimentaire locale.

\subsection{Le projet alimentaire territorial}

\subsubsection{Le dispositif et les différentes phases d'élaboration du PAT}

Figure 3 : Historique du déroulement du projet alimentaire territorial de ToursMétropole (Biais et Souchon, 2018, p. 51)

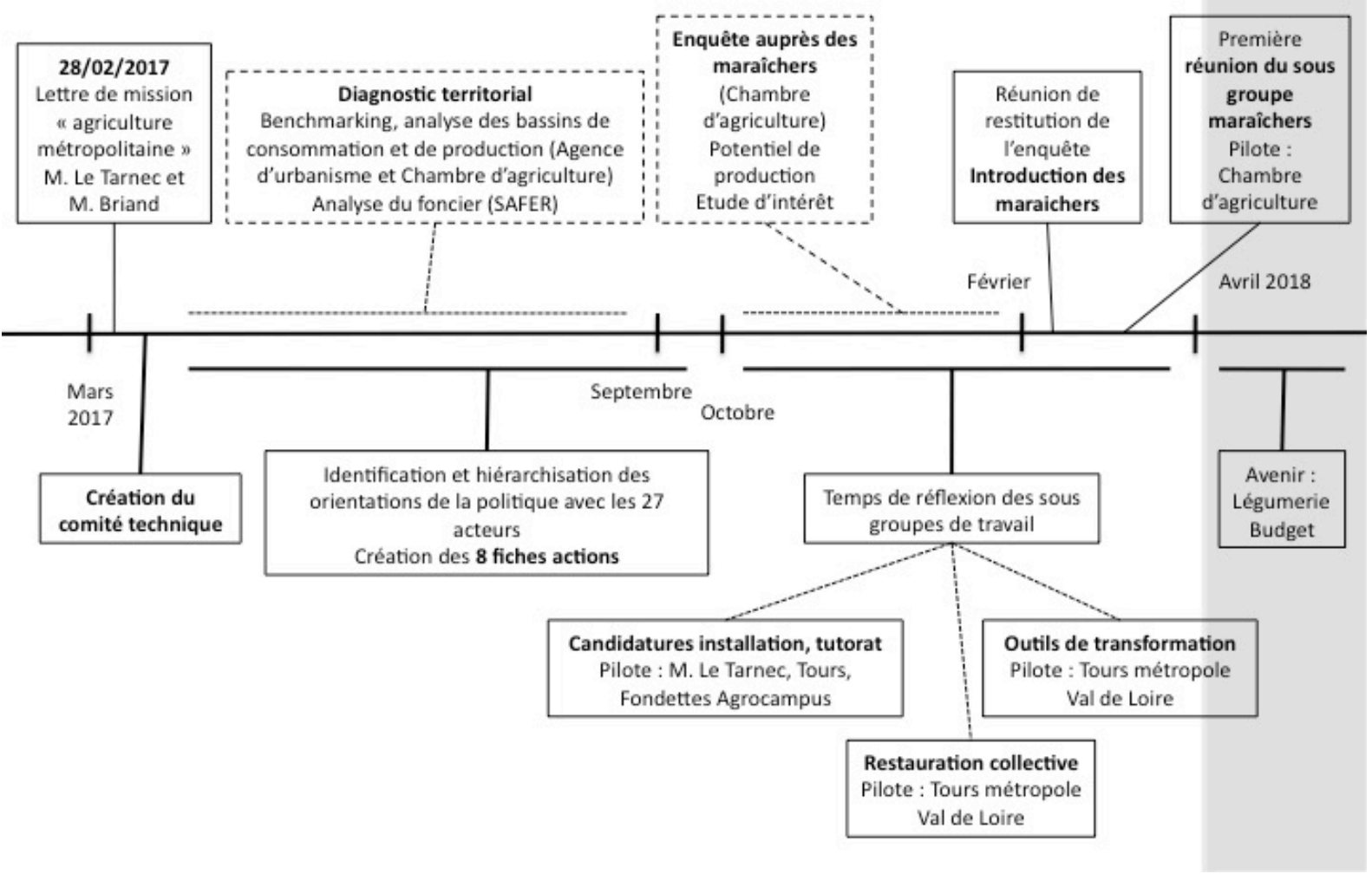

L'élaboration du projet alimentaire territorial démarre en mars 2017 et se déroule jusqu'en septembre 2018. Un comité de pilotage est constitué. L'agence d'urbanisme de l'agglomération de Tours et les directions du développement durable et du 
développement économique de la métropole constituent le groupe technique chargé d'animer les ateliers et de réaliser un diagnostic du territoire.

De mars à septembre 2017, une série de cinq ateliers a permis de confronter des points de vues parfois divergents afin d'identifier et de hiérarchiser des priorités avec comme ambition la mise en place d'un PAT métropolitain (cf. Figure 3). En partant d'une consultation générale visant à déterminer les attentes et perceptions des membres du groupe, le travail s'est, au fil de cinq séances, affiné (cf. Infra). La première séance a servi à doter le groupe d'un langage commun et à exprimer les rôles de chacun et les objectifs et actions souhaitables. Les définitions de circuits courts ou de proximité variaient. Un consensus s'est dégagé pour la proximité ; elle est limitée à une distance de $15 \mathrm{~km}$ pour le maraichage et de $80 \mathrm{~km}$ pour la production de viande. Le périmètre est centré sur l'agglomération tourangelle qui concentre le bassin de consommation (cf. carte 1). La limite de $15 \mathrm{~km}$ sera conservée tout au long des ateliers et ne sera plus débattue. Les séances 2 à 5 servent à affiner et à prioriser les objectifs jusqu'à les traduire en huit objectifs opérationnels (fiches actions ${ }^{6}$ ). La sélection finale des huit fiches actions prioritaires est issue d'un vote par les membres du comité de pilotage.

En parallèle de ces ateliers, des études sont menées par l'agence d'urbanisme et la chambre d'agriculture 37. En se basant sur l'objectif d'autosuffisance alimentaire énoncé par Tours Métropole, l'agence d'urbanisme prend l'initiative d'une étude pour mesurer la demande en légumes de l'ensemble de la population de Tours Métropole et apprécier la surface agricole nécessaire à sa production. Avec la même méthode, l'agence d'urbanisme calcule la surface nécessaire pour approvisionner les cantines scolaires et des collèges (cf. tableau 1) (Agence d'Urbanisme de l'Agglomération de Tours, 2017b). La chambre d'agriculture a conduit une enquête maraîchère pour cerner l'offre de légumes. Ce travail a beaucoup évolué au cours des discussions du groupe projet partenarial. L'étude réalisée a mis en avant une offre de produits locaux insuffisante pour répondre à la demande croissante issue des différents pôles de consommation. Les discussions ont fait sortir des avis divergents quant aux raisons de ce manque d'offre et le consensus s'est engagé autour de la formalisation d'objectifs opérationnels (cf. fiches actions ${ }^{7}$ ). La mesure la plus innovante pour la collectivité correspond à la mise en place d'un outil de transformation (légumerie) destiné à faire rencontrer l'offre des maraîchers locaux avec la demande des cuisines centrales.

La seconde phase de la démarche s'est tenue de septembre 2017 à septembre 2018. Elle était animée par les techniciens de la métropole et par l'agence d'urbanisme. Elle s'est organisée avec trois sous-groupes et est apparue comme une phase de mobilisation des forces vives en vue de la réalisation du PAT. Chaque sous-groupe de travail avait comme mission la déclinaison d'un des objectifs visant à augmenter le potentiel de production local et à organiser l'adéquation entre les producteurs et les consommateurs.

\footnotetext{
${ }^{6}$ Agir sur le foncier (renforcer le partenariat avec la SAFER, constituer un établissement public foncier, diversifier les modes d'installation), favoriser l'installation d'agriculteurs (constituer un guichet unique, développer le tutorat, dégager un budget d'aide à l'installation et au logement), assurer des débouchés multiples locaux (diversifier et centraliser la production locale pour répondre à la commande publique, mettre en place un programme pluriannuel d'investissement pour des outils de transformation).
} 
Le dispositif a évolué en deux phases en reprenant les étapes classiques de la conduite de projet. La première phase est destinée à faire partager un diagnostic. Elle se déroule en plénières au cours desquelles les acteurs sont invités à partager leurs réflexions. Des diagnostics pour éclairer les débats sont décidés puis présentés. Rapidement, la réflexion est orientée vers la restauration collective, le maraichage et les circuits courts. Les études de l'agence d'urbanisme aboutissent à la mise en lumière d'un déséquilibre entre la demande et l'offre. La réflexion n'est pas approfondie sur la compréhension de ce déséquilibre et sur le manque de compétitivité de la production maraichère locale. Les avis divergent. Néanmoins, le groupe s'engage dans la phase suivante du projet: l'élaboration d'un plan d'actions. Les actions prioritaires sont identifiées et le dispositif est reconfiguré en sous-groupes. La composition des groupes est décidée en fonction des expertises et des compétences afin de gagner en efficacité et en pertinence.

\subsubsection{Les positions des acteurs interviewés}

L'enquête maraichère menée par la chambre d'agriculture montre qu'il existe deux modèles d'exploitations sur le territoire métropolitain :

- " les maraîchers diversifiés » qui cultivent une grande diversité de produits en petits volumes sur des petites à moyennes surfaces et qui s'orientent vers la vente directe, les paniers ou les marchés ;

- " les légumiers » qui cultivent une faible diversité de produits, mais en gros volumes sur des surfaces moyennes à grandes et qui vendent généralement en gros (marché de gros, grossiste, grandes et moyennes surfaces).

Ces deux types sont présents dans le dispositif d'élaboration du PAT.

"On est parti sur une petite surface avec peu de mécanisation et puis une grande variété de légumes. 70 espèces avec plusieurs variétés ce qu'on ne retrouve pas forcément chez d'autres collègues. (...) Le projet alimentaire territorial fait partie de mes finalités. Dans mon projet, c'est de participer à l'autonomie alimentaire de mon territoire. Donc là, pour nous, ce serait concrètement par la vente directe. (...) C'est un projet à mener avec des collègues, avec un nouvel associé. C'est intéressant au niveau relationnel. C'est un projet en conformité avec nos finalités. Donc pourquoi pas? Mais on ne mise pas forcément làdessus. On ne vient pas en conquérant, en disant : il y a un nouveau marché, il faut qu'on le saisisse. On est lucides. Vu comment on travaille, on ne peut pas forcément en tirer grandchose, c'est des volumes importants. Ce n'est pas le créneau sur lequel on est. Ce n'est pas adapté à notre modèle. (...) Peut-être qu'on n'a rien à y faire et on le saura vite. Mais pour l'instant on a l'impression de participer à un projet collectif. Après si ça tourne à quelque chose de trop commercial, trop rigide, voilà on quittera le groupe. (...) L'objectif de la prochaine réunion entre maraîchers c'est de vérifier quels sont les objectifs que l'on partage. » Maraicher biologique, 7 mars 2018.

Ce maraîcher diversifié adhère au principe de fournir la restauration collective de son territoire mais il pointe le risque de décalage entre ses capacités de production et les besoins d'une cuisine centrale comme celle de Tours. Il pose les conditions de sa participation, à savoir que le projet alimentaire doit être compatible avec le fonctionnement de son exploitation. Ouvert à cette opportunité, il n'en est pas dépendant car il dispose déjà de son propre circuit de commercialisation. 
"Ce sur quoi on peut agir, c'est la restauration collective, quand on voit les quantités demandées, on peut toutes les produire sans problèmes. (...) On a tous une marge de progression. (...) Mais globalement des quantités comme ça, n'importe qui peut les faire demain sans que ça ait un impact sur sa production. Faut pas croire qu'on est au taquet sur ce qu'on produit. On est toujours à la recherche de nouveaux contacts et débouchés (...) J'ai dit à Monsieur Le Tarnec [vice président de Tours métropole et chargé de l'élaboration du PAT], faites attention à ce que vous faites parce qu'il y a déjà des acteurs qui sont en place, qui ont une expertise et qui savent faire. Il faudrait pas qu'il y ait une distorsion de concurrence » Maraicher conventionnel, 16 mars 2018.

Pour ce maraicher conventionnel, le PAT représente une opportunité de développement commercial et il se dit partant. Il préfère que le projet favorise la croissance des exploitations existantes plutôt que l'installation de nouveaux producteurs, ce qui le distingue du modèle des exploitants diversifiés. En se référant à l'histoire de la filière maraichère locale qui s'est littéralement effondrée en une trentaine d'années, ce maraîcher est très attentif à l'équilibre du marché et redoute une situation de surproduction locale qui conduise à la pénalisation des exploitations existantes. Le PAT doit s'adapter au tissu de producteurs existants et les favoriser.

"C'est assez complexe, on vient tous de différents bords avec différentes sensibilités. Inpact 37 est reconnue et appréciée par les élus et les salariés de Tours Métropole. Le message n'est pas clair, ils ne se positionnent pas sur le " bio ou pas bio ». Jacques Le Tarnec [vice président de Tours métropole et chargé de l'élaboration du PAT] veut inclure toutes les agricultures même s'il dit qu'il fera en sorte qu'il y ait une tendance vers le bio. » Technicien Inpact 37, 13 mars 2017.

Inpact $37^{7}$ souhaite rester fidèle à son modèle d'agriculture paysanne et que le PAT se traduise par l'installation de nouveaux maraîchers plutôt que par la croissance des exploitations existantes. L'association poursuit un objectif à forte valeur sociale. Elle pense que le statut quo installé lors de la phase de réflexion du PAT aboutira à une répartition des crédits entre la chambre d'agriculture et Inpact 37 de manière à contenter toutes les parties.

"À la deuxième réunion où il y avait tous les exercices avec les post-it, il y avait déjà eu des thématiques pré-identifiées sur lesquelles on a travaillé. L'idée était de cibler les actions prioritaires. Pour moi c'était un exercice intéressant parce qu'autour de la table il y avait une grande diversité de personnes. Sur l'agriculture, en tout cas, il y avait une représentation de toutes les mouvances qu'on peut avoir sur le territoire donc je pense que pour ça c'est intéressant. (...) La chambre d'agriculture est un acteur incontournable sur le sujet. (...) Bon, moi je ne pense pas qu'il faille trancher parce qu'un outil de transformation [la légumerie] peut faire les deux à partir du moment où les process sont bien séparés. Il faut que cet outil puisse répondre aux différents acteurs qui sont autour de la table. Je pense que le monde des maraichers va être très vigilant sur cet aspect " bio » parce qu'ils sont pas tous bio. Je pense qu'il faut leur laisser la possibilité d'y venir par eux-mêmes mais

\footnotetext{
${ }^{7}$ Inpact 37 (initiatives pour une agriculture citoyenne et territoriale). Cette association a pour objectif de promouvoir et de participer au développement des différentes formes d'agricultures alternatives en touraine : agriculture biologique, paysanne, citoyenne et territoriale. C'est une émanation de la confédération paysanne (www.inpact37.org)
} 
pas l'imposer. (...) De toute façon la chambre de par son modèle elle est forcément représentative de l'ensemble des agriculteurs. Après, on peut l'accuser de tout ce qu'on veut et dire qu'elle est plus tendance FNSEA, mais il n'empêche qu'elle représente tous les acteurs. » Technicien chambre d'agriculture, 6 mars 2018.

Durant la première phase de réflexion, l'animation était assurée par l'agence d'urbanisme et la chambre d'agriculture ne pouvait pas conduire les débats. Elle s'est satisfaite de l'ouverture faite à tous les modèles d'agriculture autrement dit à l'abandon de la priorité donnée à l'agriculture biologique par les élus locaux de la mandature précédente ${ }^{8}$. Vivant la présence d'Inpact 37 comme une concurrence, la chambre d'agriculture cherche à se poser comme la représentante privilégiée des intérêts des maraichers. Elle met en avant son caractère historique.

"Parce que la métropole ce n'est pas le ministère de l'agriculture, donc si elle veut créer un projet agricole elle doit le faire en lien avec les acteurs qui sont déjà dans ce domaine et qui sont beaucoup plus implantés et opérationnels. Alors ça peut paraître évident mais cela ne l'était pas au départ. Les relations entre la métropole, l'agence d'urbanisme et la chambre d'agriculture n'étaient pas au plus beau. La SAFER (...), Inpact 37, le groupement d'agriculteurs bio, (...), une centrale d'achat de traitement de produits, la confédération paysanne (...). Il y avait 27 personnes, autant vous dire que je ne faisais pas mon fier en animant ma première réunion (...) Je me disais plutôt mais comment ça va se passer ? Parce que si chacun reste sur son pré-carré en comparant ses pratiques à celles des autres qui sont toujours forcément moins bonnes ça va être difficile. (...) Alors quand j'ai vu arriver sur le tableau les premiers post-it avec écrit " arrêtons d'opposer les différents types de pratiques agricoles ", là, j'ai soufflé et me suis dit c'est bon! ça y est, ils ont compris et on va pouvoir travailler ensemble. » Vice président Tours métropole, 15 mars 2018.

Les élus souhaitent avoir une action transversale sur l'ensemble de la filière et essayent de rassembler les différentes catégories d'acteurs concernés par la mise en œuvre d'un circuit court. Ils décident de mettre en œuvre un comité technique qui sera chargé d'animer la réflexion et de faire avancer le projet. Ce comité technique n'est pas dominé par les acteurs agricoles mais par les élus locaux, leurs services et l'agence d'urbanisme. La chambre d'agriculture et la confédération paysanne (à travers l'association Inpact37) sont impliquées dans le dispositif de réflexion et parfois d'animation mais ils n'ont pas la main sur la construction et la direction de l'ensemble du dispositif. Néanmoins, le viceprésident sait qu'il a besoin d'intermédiaires or les relations avec la chambre d'agriculture sont brouillées depuis que la communauté d'agglomération Tour(s)plus a privilégié l'installation de trois maraichers en agriculture biologique en s'appuyant sur Inpact 37.

«Symboliquement, il y a la chambre et Inpact37 qui défendent deux modèles d'agriculture différents mais maintenant, on se rend compte que la chambre fait beaucoup évoluer son discours là-dessus (...) Après faut pas idéaliser non plus. Ma vision était tellement de voir

\footnotetext{
${ }^{8}$ Avant de devenir Tours-Métropole-Val-de-Loire, la communauté d'agglomération de Tour(s)plus s'était engagée dans le soutien à l'installation de maraichers. Ce qui s'est traduit par trois installations. Bien que ce ne soit pas une exigence de la communauté d'agglomération, les trois maraîchers installés se sont convertis à l'agriculture biologique. La chambre d'agriculture 37 a vécu cette opération comme un ostracisme de la part d'une agglomération marquée alors à gauche.
} 
deux mondes séparés que de la voir autour de la même table, pour moi c'est vraiment une grande victoire. Enfin victoire, c'est peut-être exagéré mais disons un pas en avant important. (...) Aujourd'hui on a réussi à mettre tout le monde autour de la table pour qu'il $y$ ait échanges, discussions et production. Donc, l'étape suivante ce n'est pas de remettre de la crispation. » Technicien Agence d'urbanisme de Tours, 14 mars 2018.

Les divergences entre la chambre d'agriculture et Inpact 37 sont patentes mais les objectifs de réunir l'ensemble des acteurs de la filière et d'avoir une action coordonnée sur l'ensemble de la filière sont les plus forts. Si bien que les discussions visent à être inclusives en faisant exprimer les attentes de chacun et en cherchant les articulations possibles. Les écueils sont clairement identifiés mais les décisions susceptibles de cliver les groupes de réflexion sont éludées et reportées en laissant planer la possibilité que tous les acteurs aient une place dans le PAT.

\subsubsection{Le contenu du PAT}

La restauration collective et le maraîchage ont été définis comme prioritaires par le groupe projet partenarial. Tout d'abord, la restauration collective est un outil facilement mobilisable par la métropole, elle peut permettre de développer et de relocaliser une partie de l'agriculture nourricière tout en étant vecteur de développement économique local. Ensuite, le maraîchage est, quant à lui, apparu comme un secteur particulièrement touché par la crise agricole. En effet face à la concurrence internationale la filière maraîchère n'est plus compétitive. La réflexion s'est orientée vers la mise en relation de l'offre et de la demande locale à travers un outil de transformation : une légumerie. Les études menées visaient à quantifier l'offre et les besoins et à dimensionner la légumerie.

La restauration des écoles et des collèges inclut déjà $20 \%$ de légumes locaux. Deux scénarios d'approvisionnement ont été étudiés : la part des produits locaux est portée jusqu'à $100 \%$ (scénario 1) et la part des produits locaux est portée jusqu'à $50 \%$ (scénario 2$)^{9}$.

Tableau 1 : Quantités de légumes à produire pour la restauration des élèves du primaire et du collège de Tours métropole

\begin{tabular}{|c|c|c|c|}
\hline Scénario 1 (100\% local) & $\begin{array}{lr}\text { Quantités } & \text { de } \\
\text { légumes } & \text { bruts } \\
\text { nécessaires } & \text { (en } \\
\text { tonnes }(\mathrm{t})) & \end{array}$ & $\begin{array}{l}\text { Surfaces de maraichage } \\
\text { pour les produire (en } \\
\text { hectares (ha)) }\end{array}$ & $\begin{array}{lr}\text { Nombre de } \\
\text { maraichers à } \\
\text { installer }\end{array}$ \\
\hline $\begin{array}{l}\text { Pour les écoles maternelles et } \\
\text { primaires }\end{array}$ & 260 & 18 & \\
\hline Pour les collèges & 155 & 9,6 & \\
\hline Total & 415 & 27,6 & 12 \\
\hline Scénario 1 (50\% local) & $\begin{array}{lr}\text { Quantités } & \text { de } \\
\text { légumes } & \text { bruts } \\
\text { nécessaires (en t) }\end{array}$ & $\begin{array}{l}\text { Surfaces de maraichage } \\
\text { pour les produire (en } \\
\text { ha) }\end{array}$ & $\begin{array}{lr}\text { Nombre de } \\
\text { maraichers à } \\
\text { installer }\end{array}$ \\
\hline
\end{tabular}

\footnotetext{
${ }^{9}$ Bien que les collèges dépendent de la compétence du département, ce dernier n’a pas été associé au comité de pilotage du PAT.
} 


\begin{tabular}{|l|c|c|c|}
\hline Pour les écoles maternelles et & 97,5 & 6,75 & \\
primaires & 58,1 & 3,6 & \\
Pour les collèges & $\mathbf{1 5 5 , 6}$ & $\mathbf{1 0 , 3 5}$ & $\mathbf{5}$ \\
\hline
\end{tabular}

D’après Agence d'Urbanisme de l'Agglomération de Tours, 2017b

Après une étude fine sur le contenu de l'assiette d'un repas de collégien et celui d'un écolier, les besoins en tonnage et en superficie de production ont été identifiés en tenant compte de l'estimation de produits locaux déjà utilisés (soit environ 20\%) dans la restauration collective actuellement. Sur la base d'une surface de 2,25 ha nécessaires pour une exploitation maraichère, le calcul aboutit à la nécessité de trouver, ou installer, douze (scénario 1) ou cinq (scénario 2) maraichers qui dédieraient 100\% de leur production à la restauration des élèves.

L'enquête sur l'offre des producteurs a été menée par la chambre d'agriculture qui possède les coordonnées de ces derniers. Dans un rayon de quinze kilomètres autour de l'agglomération, une vingtaine de maraichers, dont quatre sont en agriculture biologique, a été identifié. Cette enquête des maraichers montre que quatorze maraichers sont intéressés par la restauration collective mais font état de nombreux freins : nécessité d'un outil de transformation, planification, organisation logistique, prix sécurisés et justes. Les préoccupations mentionnées par les producteurs sont en adéquation avec les actions identifiées par le groupe projet partenarial.

En ce qui concerne la légumerie, un groupe de travail a été dédié et a commandité une étude de faisabilité auprès d'un prestataire. Celui ci a dessiné une légumerie capable de traiter les besoins en légumes des écoles de la métropole. Le seuil de rentabilité de la légumerie est estimé à 300 tonnes de légumes traités/an. Elle est agencée pour pouvoir traiter séparément les légumes issus de l'agriculture biologique et conventionnels. Un protocole d'engagement a été rédigé pour préciser les conditions de livraison des légumes à la légumerie et dans lequel sont rappelés les objectifs d'atteindre au minimum $50 \%$ de produits locaux et $20 \%$ de produits biologiques dans la restauration collective.

En septembre 2018, une synthèse des travaux menés par les trois sous-groupes de travail a fait l'objet d'une assemblée plénière. Le regret, clairement exprimé lors de cette assemblée par certains acteurs concernés, est l'absence d'affichage de production biologique comme l'axe principal du PAT. Toutefois, si cette ambition n'est pas affirmée pour ce qui concerne le PAT, elle reste implicite pour la politique d'aide à l'installation de maraichers telle qu'elle a été initiée du temps de la communauté d'agglomération de Tour(s)plus et poursuivie par Tours Métropole. 


\section{Quelle portée novatrice pour les projets alimentaires territoriaux ?}

\subsection{Circuits de proximité et circuits longs}

\subsubsection{Un projet agri-alimentaire}

Une enquête menée par le RNPAT (2018) distingue deux grandes conceptions de projets alimentaires territoriaux: les projets agri-alimentaires et les projets systémiques ou transversaux.

Les projets agri-alimentaires sont des outils pour des projets sectoriels. Ils privilégient des mesures opérationnelles et à court terme. Le RNPAT les qualifie de "ponctuels » lorsqu'ils sont initiés par des acteurs agricoles et centrés sur des objectifs économiques. Ils sont dits «structurants » lorsqu'ils portent sur le lien production/alimentation. Dans ce cas, l'orientation économique est forte mais le projet est ouvert à d'autres acteurs.

Les projets systémiques sont élaborés avec la société civile. Les enjeux économiques ne sont pas forcément dominants. Ce sont des outils d'une politique territoriale en faveur de l'alimentation. Ils définissent une stratégie et une concrétisation à moyen terme.

La grande majorité des PAT sont de type agri-alimentaire et visent à structurer une économie agricole et ses filières en créant de la valeur ajoutée. Le PAT de Tours rentre dans cette catégorie. La légumerie est destinée à structurer la filière c'est-à-dire à centraliser l'offre et à transformer les produits pour les rendre utilisables par les cuisines centrales. Cela revient à réserver un débouché pour les producteurs. Parallèlement, la métropole tourangelle poursuit ses efforts de soutien à l'installation de nouveaux maraichers afin d'augmenter l'offre locale.

Pour le RNPAT, le contenu du projet alimentaire territorial dépend fortement de la méthode pour l'élaboration du diagnostic. Il constate que les projets agri-alimentaires sont élaborés sur la base d'expertises techniques serrées et pointues qui sont orientées vers des objectifs opérationnels. Comme dans le cas de Tours Métropole, l'analyse se limite à la quantification de l'offre et de la demande et à leur mise en relation. Ce type de projet débouche sur des actions rapides, précises et pertinentes.

\subsubsection{La permanence du poids des circuits longs}

La progression des circuits de proximité est à replacer dans une tendance plus générale d'offre de modèle de développement. Baysse-Lainé et Perrin (2017) constatent une relocalisation indéniable de la production alimentaire. Néanmoins ces auteurs estiment qu'elle ne dépasse pas $6 \%$ du poids des produits alimentaires consommés par les habitants d'une ville comme Millau et qu'elle occupe au plus $15 \%$ de la surface agricole utile autour de Millau. Pour Paris, Bognon (2017) estime que les volumes de produits alimentaires de proximité sont insuffisants pour constituer un substitut significatif aux circuits d'approvisionnement conventionnel. Les circuits courts ne remplacent pas véritablement les circuits conventionnels bien qu'ils se multiplient et soient en vogue. Une comparaison des projets alimentaires de Paris et de Brive avait montré les mêmes limites (Bognon et Marty, 2015). Les circuits courts reposent sur la qualité et la proximité. Leur fonctionnement est une rupture par rapport à l'organisation des filières 
longues qui ont une logistique et des techniques adaptées à la spécialisation et aux grandes distances. Les initiatives de Paris et de Brive ne changent pas en profondeur l'organisation des filières alimentaires. Pour ces auteurs, l'intérêt des ces politiques alimentaires locales encore marginales serait d'ouvrir une brèche en proposant un modèle alternatif. Leur "force » est de proposer un contre modèle pour la réflexion. Plutôt qu'opposition, il y a hybridation car le système dominant récupère les qualités des circuits de proximité pour conserver la clientèle tentée par de nouveaux modèles. Les acteurs des circuits conventionnels font preuve de pragmatisme et d'opportunisme pour adapter leurs systèmes à la marge sans renoncer à leur modèle industriel (Bognon, 2017). Le contre modèle peut aussi servir aux acteurs locaux pour négocier l'adaptation des modalités des circuits longs standardisés aux spécificités locales ce qui constitue également une hybridation sous l'impulsion des acteurs locaux cette fois (DubuissonQuellier et Le Velly, 2008).

La question est de savoir si les innovations et les différenciations portées par les circuits courts en tant que niches peuvent mettre en cause le système dominant et engager une transition? Les niches alimentaires peuvent rester des niches soit parce qu'elles n'ont pas vocation à se généraliser soit parce que le système dominant continue d'imposer ses normes et ses savoirs faire. Va-t-on vers une coalescence de modèles dispersés ou vers un maintien d'un modèle dominant qui cantonne les alternatives?

Hubert (2020) défend l'idée que la coexistence de divers modèles n'est pas suffisante pour une évolution significative des modèles et considère que c'est une impasse. En effet, le modèle alimentaire industriel est enfermé dans une logique d'augmentation du rendement et de la productivité. Le PAT de Tours Métropole rassemblant maraichers conventionnels et biologiques n'est, dans ce cas, pas dénué d'intérêt. Il ne réinterroge pas l'hégémonie des circuits longs car la question de la disponibilité du foncier « alimentaire » n'est pas posée. Ce qui dans les faits revient à ce que la place respective des circuits de proximité et des circuits longs ne soit pas questionnée.

\subsection{Un projet alimentaire territorial non circonscrit spatialement}

La clef de voûte du PAT de Tours-Métropole est la légumerie car elle permet une mise en relation des producteurs et des utilisateurs. Bien que l'équipement d'une légumerie ait été débattu collectivement au sein du PAT, la collectivité est l'acteur central du projet. Elle est placée comme chef de file du projet alimentaire territorial et à ce titre elle en a eu non seulement l'initiative mais aussi la responsabilité de configurer le dispositif de gouvernance et de dimensionner les actions opérationnelles.

L'alimentation est un élargissement du champ d'intervention de la collectivité. Auparavant les collectivités agissaient en faveur des producteurs. En optant pour la relocalisation, les collectivités mobilisent deux leviers; la restauration collective et la transformation. Elles structurent l'offre, formalisent la demande et assument les infrastructures intermédiaires. C'est un changement de rôle car la transversalité de la question alimentaire les amène à une coordination horizontale entre des acteurs locaux aux intérêts divergeant. Ce rôle de régisseur de territoire bouleverse l'action des collectivités (Cresp, 2018). En matière économique, elles avaient un rôle de soutien indirect. De facilitateur, elles deviennent maître d'ouvrage. 
Les objectifs de la loi pour atteindre $50 \%$ de produits locaux et $20 \%$ de produits biologiques doivent être atteints en 2025, c'est-à-dire à brève échéance. Les calculs montrent qu'il faudrait installer cinq maraîchers d'ici 2025 sur une dizaine d'hectares (cf. tableau 1), le double si l'on tient compte du seuil de rentabilité de la légumerie. Dans son étude l'agence d'urbanisme (2017b) a calculé que 16000 hectares sont potentiellement disponibles pour la production alimentaire. Ce chiffre s'élève à 50000 hectares si l'on considère le rayon de $15 \mathrm{~km}$ fixé par le comité de pilotage du PAT. Bien qu'installer cinq maraichers représente du temps et peut paraître un objectif difficile pour 2025, la ressource foncière est suffisamment abondante pour que Tours métropole puisse fournir la légumerie. Baysse-Lainé et Perrin (2017) constatent que la relocalisation de la production alimentaire ne conduit pas à l'émergence de nouvelles ceintures maraichères identifiées car le foncier "alimentaire » est dispersé dans un bassin de production majoritairement tourné vers les marchés internationaux. De plus, le foncier alimentaire semble se relocaliser dans les parcelles les moins productives ou les plus exposées à des risques naturels. Cette dynamique foncière révèle la fragilité de la relocalisation de la production alimentaire et le déséquilibre avec les producteurs en circuits longs pour la captation du foncier. Enfin le cahier des charges pour l'approvisionnement de la légumerie ouvre le périmètre de provenance des produits alimentaires. Fixé à $15 \mathrm{~km}$ pour les légumes, il peut être étendu jusqu'aux départements limitrophes ${ }^{10}$.

$\mathrm{Au}$ final, la substitution progressive des producteurs des circuits longs par les producteurs de circuits courts n'a pas lieu. Le comité de pilotage a fixé une limite de 15 $\mathrm{km}$ qui dilue le foncier alimentaire sur un territoire si vaste que la question du remplacement d'un filière par une autre ne se pose pas.

\subsection{L'absence de réflexion sur la qualité de la proximité et les limites du consensus}

Pour le président de Tours métropole, le PAT a pour ambition «d'assurer une forte part d'autosuffisance alimentaire dès 2025. (...) j'ai souhaité la mise en œuvre de trois axes forts et concrets pour rendre visible et lisible ce projet: fédérer les acteurs du secteur alimentaire, accroître sensiblement la production et la consommation de proximité, promouvoir une alimentation saine et responsable ${ }^{11}$. Cet objectif peut apparaître comme un mythe ou une utopie compte tenu de la très faible part des circuits courts dans l'alimentation de villes même moyennes (Baysse-Lainé et Perrin, 2017). Il n'en demeure pas moins qu'il va orienter les efforts des acteurs pour relocaliser la production. Or l'objectif d'autosuffisance alimentaire ne définit pas la plus-value écologique, nutritive, sociale des produits alimentaires dits de proximité (Fleury et Vidal, 2010). Au contraire, elle peut réduire le problème à une approche quantitative : augmenter les volumes de produits "proches» ce qui ne remet pas en cause, voire conforte le modèle d'une agriculture industrielle basée sur les gains technologiques (Hubert, 2020)

\footnotetext{
10 Tours-Métropole-Val-de-Loire, 2019, Projet alimentaire territorial de Tours-Métropole-Val-de-Loire. Protocole d'engagement pour une restauration collective de proximité et de qualité, 3 p.

11 Président de Tours-Métropole-Va-de-Loire, 2018, Tours métropole Le Mag, dossier la métropole versant agricole, $n^{\circ} 62$, p. 3
} 
Le RNPAT (2018) identifie deux grands types d'expertises privilégiées par les collectivités locales: une expertise de démarche et une expertise technique. Pour l'expertise de démarche, la collectivité concentre son effort sur le processus d'élaboration du PAT. Il s'agit d'ouvrir la démarche aux parties prenantes et à la population. Cette démarche peut être périlleuse pour la collectivité. L'implication des citoyens favorise l'expression des besoins sociaux qui peuvent remettre en cause les valeurs du système agroindustriel. Mal maitrisée cette démarche peut aboutir à un départ des acteurs économiques de poids du projet et à la réduction de la réflexion à une sphère militante à l'impact marginal. À l'inverse, une concertation de façade peut conduire à un désintérêt pour les mesures adoptées qui ne trouveront pas de relais. Pour le RNPAT, cette posture nécessite de considérer la conduite du processus comme un champ d'investissement en soi par la collectivité. Ce type d'expertise est adapté aux PAT systémiques. Pour les PAT agri-alimentaires, c'est l'expertise technique qui est privilégiée. La gouvernance est moins ouverte et le périmètre est plus restreint. Le diagnostic se limite à une analyse de l'offre et de la demande et à leur mise en relation. Les impératifs de la crise agricole, la pression professionnelle et la recherche de solutions opérationnelles à court terme restreignent la réflexion sur la qualité de l'alimentation.

En l'absence de réflexion sur la qualité, le problème à résoudre reste quantitatif. Où trouver les légumes pour remplir les objectifs quantitatifs de la loi ? La collectivité peut augmenter la production maraichère ou recruter des producteurs qui vont livrer à la légumerie. L'installation de nouveaux maraîchers est une solution de moyen terme. La métropole de Tours poursuit l'action initiée du temps de la communauté d'agglomération. Un quatrième maraicher est en passe d'être installé à quelques kilomètres de Tours. L'étude de l'agence d'urbanisme avait montré qu'il fallait cinq maraîchers qui consacrent $100 \%$ de leur production à la légumerie pour atteindre l'objectif de $50 \%$ de produits locaux. L'augmentation des surfaces par substitution des surfaces dédiées aux circuits longs par des surfaces dédiées aux circuits courts ne fonctionne pas (cf. 3.2). Il reste pour le groupe de réflexion sur le PAT la solution de trouver des candidats, des producteurs installés pour fournir les cantines via la légumerie.

La collectivité peut s'appuyer sur des têtes de réseaux traditionnels ou nouveaux (chambre de commerce, chambre des métiers, comité communal d'action sociale ...). Dans le cas de Tours, elle n'a impliqué que la chambre d'agriculture et Inpact37 qui ont un lien direct avec les acteurs du secteur qu'elle veut mobiliser : les agriculteurs. Naves (2016) montre que pour les chambres d'agriculture, les circuits courts ou de proximité sont à contre-courant du modèle de développement intensif et spécialisé ; de fait, les chambres d'agriculture manquent de références techniques pour les encourager. Une situation qui les affaiblit d'autant plus que ce thème qui est à l'agenda politique amène les collectivités à intervenir de plus en plus sur les questions concernant l'agriculture. La chambre d'agriculture 37 est dans ce cas de figure. Le développement des circuits courts l'a prise au dépourvu. Mais concurrencée par d'autres acteurs, comme Inpact 37, pour qui les circuits courts pour les petites exploitations sont une voie de développement, elle a cherché à se positionner comme intermédiaire central par rapport à la collectivité. Situation qui fait que la chambre d'agriculture 37 n'a eu de cesse de revendiquer son rôle historique et de représentant de tous les agriculteurs. Elle a utilisé cet argument 
pour écarter l'idée d'une spécialisation du PAT dans le bio en arguant une ouverture à l'ensemble des producteurs. Forte de son carnet d'adresses, elle s'est placé en intermédiaire précieux et incontournable en se chargeant de mener les études auprès des maraichers. L'implication de la chambre d'agriculture dans le PAT a été un soulagement pour la collectivité qui se trouvait démunie pour travailler avec les agriculteurs.

Un projet territorial vu comme un système dynamique complexe reposant sur des relations de partenariat amène les acteurs à construire des solidarités. Ce sont elles qui permettent de partager les ressources et l'appropriation commune d'un espace. Cette construction territoriale amène à créer un dedans et un dehors. Quel que soit le contenu du projet, elle définit des conditions d'appartenance qui sont donc excluantes pour ceux qui ne les remplissent pas. C'est précisément l'inverse de ce qui a été recherché. La collectivité était à la recherche d'un projet inclusif. Elle a trouvé la chambre d'agriculture comme allié objectif. En écartant les questions qui clivent : bio/pas bio et le sens donné au «local», elle s'est privée d'une réflexion sur les enjeux et la valeur ajoutée (pas seulement économique) du local. La collectivité s'empêche ainsi du pouvoir d'orientation que lui donne un équipement de transformation à la jonction entre la production et la consommation. Les objectifs de consensus sont devenus prioritaires et formels. L'objectif n'a pas été de faire émerger une vision du territoire propre au collectif mais de partager unanimement un projet. Le projet alimentaire de ToursMétropole est davantage un projet alimentaire localisé que territorial. Le PAT est une occasion manquée pour générer un développent territorial en usant de l'alimentation comme levier tel que défini par Loudiyi et Houdart (2019). En effet la venue de nouveaux acteurs sur la question alimentaire n'est pas une innovation en soi. L'innovation repose la délimitation et l'activation d'une ressource spécifique à travers le mode de coordination des acteurs. En ayant fait l'impasse sur le lien entre qualité alimentaire et ressources locales, le PAT de Tours métropole est resté sur des ressources substituables et délocalisables.

\section{Conclusion}

Cette phase d'élaboration du PAT peut être vue comme une phase de mobilisation des acteurs et de construction de la confiance. Des diagnostics sont présentés pour construire une vision partagée du territoire. Les réunions sont animées afin que tous les acteurs s'expriment et cernent les priorités. Assez rapidement les trois priorités qui se dégagent : installer des maraichers, viser la restauration collective, installer une légumerie sont finalement classiques et font consensus. Le périmètre de quinze kilomètres pour les maraichers est fixé par le groupe lui-même, sans que soient précisés les qualités de cette proximité et de ce seuil. Les participants sont conscients de leurs différences mais ils se prêtent à cette première phase de réflexion consensuelle. Le premier objectif de la collectivité était de réunir tous les acteurs et de répondre à la loi. Les questions clivantes (proportion de bio, respect de l'environnement) ne sont pas débattues et le groupe avance sur la dimension économique du projet alimentaire territorial c'est-à-dire l'offre d'un débouché aux producteurs locaux.

La loi fixe à $20 \%$ la quantité minimale de produits biologiques dans les cantines scolaires et le PAT reprend cet objectif sans aller au-delà. L'espoir des élus est que le 
côtoiement de producteurs conventionnels et de producteurs biologiques dans un même projet conduise à une émulation vertueuse vers plus d'agriculture biologique ou du moins vers l'adoption de pratiques moins polluantes. Mais pour Muchnik et al. (2008), les modes de production biologiques et conventionnels sont clivants du fait des représentations sociopolitiques associées, de la relation au marché de producteurs et de la finalité du travail. Ce dialogue n'est donc pas une évidence. Néanmoins la restauration collective et les outils de transformation tels que les légumeries ont un potentiel d'évolution des pratiques car ils permettent de dresser des ponts entre producteurs biologiques et conventionnels, entre producteurs et consommateurs (Lamine et Cambien, 2011). Ce sont des lieux d'échanges d'informations mais aussi de services (prêt de matériel, entre-aide) qui favorisent une compréhension mutuelle et des apprentissages.

La LAAF du 13 octobre 2014 en fixant les quotas de produits locaux ou biologiques dans les restaurants scolaires ne fait pas de lien avec les ressources notamment foncières nécessaires à cet objectif. C'est précisément la démarche de projet qui devrait permettre de les trouver à travers la mobilisation des acteurs. Les métropoles sont issues de la loi de modernisation de l'action publique territoriale et d'affirmation des métropoles (Maptam) et ont un champ de compétence très large en matière d'aménagement, de développement économique et d'environnement. La LAAF les désigne chef de file du PAT et elles sont compétentes pour les documents d'urbanisme (SCOT et PLU) ce qui devrait leur permettre de lier production alimentaire locale avec l'identification d'un foncier nécessaire à sa production. Les lois " grenelles » et la loi Accès au Logement et Urbanisme Rénové (ALUR) encouragent les collectivités à limiter l'étalement urbain et à favoriser la densification mais elles ne posent pas la question de la destination des productions issues des terres ainsi épargnées de l'artificialisation. Le foncier maraicher est dilué dans un vaste bassin de production tourné vers les marchés internationaux, le foncier n'apparaît pas comme un enjeu mais comme un ressource abondante en dépit des incitations à sa protection (Serrano et Vianey, 2014). L'articulation entre la loi agricole et la réglementation foncière n'est pas faite.

Le projet alimentaire déstabilise les acteurs dans leurs rôles traditionnels. Les collectivités abordent un champ qui leur est méconnu et se retrouvent au milieu d'acteurs aux intérêts divergents. Les chambres d'agriculture ont l'impression de perdre leur monopole et leur expertise. Dans cet espace ouvert d'élaboration d'une nouvelle politique, les habitants peuvent être vus comme le problème mais aussi comme la solution. Les habitants en remettant en cause le système agroindustriel accroissent la déstabilisation des autres acteurs. Mais comme les autres, ils peuvent acquérir plus de compétences, d'autonomie (empowerment) et c'est finalement le système complet d'acteurs impliqué dans le projet alimentaire qui acquiert plus de pouvoir pour décider par lui-même (Chiffoleau et Prevost, 2012).

L'état des lieux des projets alimentaires territoriaux dressé par le RNPAT (2018) a montré la rareté des projets alimentaires véritablement transversaux. Le rôle des habitants dans la transversalité des projets apparaît éminent. Dans sa comparaisons de projets néoaquitains, Banzon et al (2019) montrent que le seul PAT initié par des citoyens est véritablement transversal et bouscule le projet de territoire qui déclasse jusque là l'agriculture locale. Ce projet était défendu par les élus locaux. Les habitants remobilisent l'agriculture comme une activité porteuse de développement en s'appuyant 
sur les contraintes environnementales locales comme une opportunité d'innovation. La diversité des PAT est inhérente à l'autonomie et à la primauté donnée aux acteurs locaux. Maréchal et al (2019) dans une étude prospective dessinent trois scénarios ${ }^{12}$ de PAT et mettent en évidence les conditions de réalisation de chacun d'entre-eux. Le PAT de Tours métropole correspond au scénario 1. Faute d'un décloisonnement des approches, le PAT se limite à un approvisionnement des cantines. Les rationalités opérationnelles et de management conduites par la collectivité dominent. La profession agricole s'implique peu. Le scénario 3 correspond à une reprise en main corporatiste de la démarche. Les acteurs agricoles et industriels privilégient les solutions technologiques reposant sur des investissements financiers. Les solutions de ruptures (exemple permaculture, produits biologiques) sont cantonnées à des niches. Dans le scénario 2, les questions de santé, cadre de vie, environnement sont au cœur du projet alimentaire qui repose sur une alliance entre la société civile et le tissu entrepreneurial local. Les collectivités sont dans une posture d'accompagnement.

La question alimentaire a un fort potentiel d'innovation car elle est multidimensionnelle et oblige à décloisonner les acteurs et les savoirs. Les solutions sont encore à inventer et dépendent des acteurs localement impliqués et de leurs ressources. Plutôt que de proposer un nouveau schéma intégrateur cherchant à prendre en compte l'ensemble des dimensions de l'alimentation et l'ensemble du système alimentaire local, Bricas et al (2017) proposent une démarche qui admet la diversité des chemins. Le projet alimentaire est construit chemin faisant par innovations, par tâtonnements, par adaptation en fonction des moyens disponibles. Pour réaliser le scénario 2, la bonne volonté des habitants ou des associations ne suffit pas. Le groupe d'acteurs et de citoyens doit posséder une forte capacité réflexive pour expérimenter et ajuster le projet. Les collectivités ont tout leur rôle à jouer en adoptant une posture d'accompagnateur et en soutenant les capacités cognitives et d'analyse du collectif.

\section{Références}

Agence d'Urbanisme de l'Agglomération de Tours, 2017a, Projet alimentaire territoriale de Tours Val de Loire. Synthèse des 5 ateliers de travail tenus entre mars et juillet 2017, Tours Métropole Val de Loire. 40 p.

Agence d'Urbanisme de l'Agglomération de Tours, 2017b, Projet alimentaire territoriale de Tours Val de Loire. Tome 2. Diagnostic territorial. Tours Métropole Val de Loire, 120 p.

Banzo M., Corade N. et Lemarie-Boutry M., (2019), «Les Projets Alimentaires de Territoire sont-ils des Projets de Territoires Alimentaires ? Une analyse à partir de trois projets néo-aquitains », 13 Journées de Recherches en Sciences Sociales, Bordeaux (en ligne) https://www.sfer.asso.fr/source/jrss2019/articles/A13_Banzo.pdf

\footnotetext{
12 Scénario 1 : "l'enlisement insitutionnel", scénario 2 : "une onstellation de démocraties alimentaires locales", scénario 3 : "il faut que tout change pour que rien ne change”
} 
Baysse-Lainé A. et Perrin C. (2017). " Les espaces agricoles des circuits de proximité : une lecture critique de la relocalisation de l'approvisionnement alimentaire de Millau », Natures Sciences Sociétés, Vol. 25, nº 1, p. 21-35

Biais C. et Souchon C. (2018). L'agriculture urbaine un levier pour la mise en œuvre de la multifonctionnalité des espaces dans les documents de planification. Mémoire de fin d'études. Tours, Polytech Tours

Bognon S. (2017). «Vers la reterritorialisation du réseau d'approvisionnement alimentaire parisien ? Trois approches de la mobilisation des proximités ». Flux, n 109 110, p. 118-128.

Bognon S. et Marty P. (2015). «La question alimentaire dans l'action publique locale. Analyse croisée des trajectoires municipales de Paris et de Brive-la-Gaillarde », VertigO la revue électronique en sciences de l'environnement [En ligne], vol. 15, $\mathrm{n}^{\circ} 2$, URL : http://journals.openedition.org/vertigo/16401

Bonny S. (2005). « Les systèmes de production agricole dans la chaîne agroalimentaire : position et évolution ", Économie rurale, $n^{\circ} 288$, p. 91-98.

Bricas N., Soulard C.T. et Arnal C. (2017) «Croiser enjeux de durabilité et leviers de politiques urbaines » in Brand C., Bricas N., Conare D., Daviron B., Debru J., Michel L. et Soulard C.T. (ED.), Construire des politiques alimentaires urbaines: concepts et démarches, éditions Quae, Versailles, p. 121-136

Chiffoleau Y. et Prevost B. (2012). " Les circuits courts, des innovations sociales pour une alimentation durable dans les territoires », Norois, ${ }^{\circ} 224$, p. 7-20.

Creps M. A. (2018). Émergence et construction des politiques alimentaires des collectivités territoriales. Étude comparative de six territoires témoins, RNPAT, Terres en Villes

Delfosse C. (2012). La France et ses terroirs. Un siècle de débats sur les produits et leurs liens à l'espace. Pour, n 215-216, p. 63-74.

Dubuisson-Quellier S. et Le Velly R. (2008). " Chapitre 8. Les circuits courts entre alternative et hybridation », In Maréchal G. éd., Les circuits courts alimentaires. Bien manger dans les territoires. Éducagri éditions, p. 103-112.

Guiomar X. (2012). " De la reterritorialisation à la relocalisation des filières, avec ou sans terroir », Pour n $^{\circ} 215-216$, p. 147-161

Fleury A. et Vidal R. (2010). «L'autosuffisance agricole des villes, une vaine utopie ? » La vie des idées, dossier "Les apories de la ville durable », www.laviedesidees.fr/Lautosuffisanceagricole-des.html.

Hubert B. (2020). «Agriculture et alimentation. Les modèles de production questionnés : l'impératif du changement agroécologique ", Raison présente, vol. $213, \mathrm{n}^{\circ}$ 1, p. 85-96. 
I.N.S.E.E. 2011. "Les aires urbaines de la région Centre s'étendent et se densifient peu." Insee Centre Info(173): 6 p.

Lamine C. et Cambien L. (2011). Les transitions vers l'agriculture biologique : une approche à l'échelle d'un système agri-alimentaire territorial, Colloque INRA Écologisation des politiques publiques et des pratiques agricoles, 16-18 mars, Avignon

Lamine C. et Chiffoleau Y. (2012). "Reconnecter agriculture et alimentation dans les territoires : dynamiques et défis », $\underline{\text { Pour }} \mathrm{n}^{\circ}$ 215-216, p. 85-95

Loudiyi S. et Houdart M. (2019), «L'alimentation comme levier de développement territorial ? Réflexions tirées de l'analyse processuelle de deux démarches territoriales », Économie rurale, vol. $367, \mathrm{n}^{\circ} 1$, p. 29-44

Maréchal G., Noel J, et Wallet F. (2018). « Les projets alimentaires territoriaux (PAT) : entre rupture, transition et immobilisme ? », Pour, $\mathrm{n}^{\circ} 234-235$, p. 261-270

Montaigu D. (2018). L'ingénierie financière des projets alimentaires territoriaux. Grenoble, Terres en Ville.

Muchnik J., Sanz-Cañada J. et Torres Salcido G. (2008). "Systèmes agroalimentaires localisés, état des recherches et perspectives », Cahiers agricultures, vol. 17, n 6, p. 513519.

Naves P. (2016). " L'encadrement des circuits courts. Du secteur agricole aux territoires? », Économie rurale, ${ }^{\circ} 355$, p. 3-19.

Perrin C et Soulard C.T. (2014). "Vers une gouvernance alimentaire locale reliant ville et agriculture. Le cas de Perpignan », Géocarrefour, vol. 89, n 1-2, p. 125-134.

Perrin C. et Soulard C.T. (2017). "Introduction. L'agriculture dans le système alimentaire urbain : continuités et innovations », Natures Sciences Sociétés, vol. 25, $n^{\circ} 1$, p. 3-6.

Praly C., Chazoule C., Delfosse C. et Mundler P. (2014). « Les circuits de proximité, cadre d'analyse de la relocalisation des circuits alimentaires ». Géographie, économie, société, vol. 16, n 4 , p. 455-478.

RNPAT (2018). Coconstruire votre projet alimentaire territorial. Repères et points de vigilance, $23 \mathrm{p}$

Serrano J. et Vianey G. (2014). « Patrimonialiser des activités agricoles pour banaliser la consommation d'espaces agricoles périurbains: réflexions à partir du cas de l'agglomération de Tours », Géographie, économie, société, vol. 16, n³, p. 297-314. 